\title{
ZUM GELEIT: \\ FRAGEN ZUR STÄDTEHANSE \\ UND STAND DER HANSEFORSCHUNG IN DER DDR \\ ANGESICHTS DES 600. JAHRESTAGES \\ DES STRALSUNDER FRIEDENS
}

\author{
von Eckhard Müller-Mertens
}

Am 24. Mai 1370 schlossen die seit 1367 mit Dänemark im Kriege stehenden Städte zu Stralsund Frieden mit der dänischen Krone. Die Kölner Konföderation wendischer und preußischer Hansestädte sowie nichthansischer Städte der Zuidersee, Hollands und Seelands - im Bunde mit den Herzögen von Mecklenburg, dem mecklenburgischen König Schwedens, den Grafen von Holstein und aufständischen jütischen Adeligen - hatte die Könige von Dänemark und Norwegen, Waldemar IV. Atterdag und Haakon VI., geschlagen. Die Hegemonialpolitik Waldemars IV. Atterdag, der ein starkes dänisches Königtum erstrebte, die ökonomische Vorherrschaft der Hanse gegenüber Dänemark beseitigen wollte, 1360 Schonen von Schweden zurückgewonnen, 1361 Gotland erobert hatte, war endgültig gescheitert.

Der Stralsunder Friede, der Kulminationspunkt in der vielhundertjährigen Geschichte der Hanse, die im 12. Jahrhundert begann, im 17. endete und als hanseatische Geschichte bis in das 20. Jahrhundert führt, jährt sich in diesem Jahre zum sechshundertsten Male. Das Jubiläum fordert eine neue Verständigung und den weiterführenden Meinungsstreit über Grundfragen hansischhanseatischer Geschichte heraus.

$\mathrm{Zu}$ den Grundfragen gehört die Frage nach dem Platz des Friedens von Stralsund im Werden und Vergehen der Hanse. Unbestritten stellt der Sieg der Kölner Konföderation über das dänische Königtum den absoluten Höhepunkt hansischer Geschichte dar. Doch steht dieser, der Stralsunder Friede von 1370, bereits am nahen Ausgang der Blütezeit der seit den letzten Jahrzehnten des 13. Jahrhunderts aus der Kaufmannshanse entwickelten Städtehanse? Oder setzt deren eigentliche geschichtliche Leistung nicht erst zu diesem Zeitpunkt großartig ein, um ein Jahrhundert der Blüte zu überdauern? Beim Nachdenken über die Definition, die politische Funktion und den Klassencharakter der Städtehanse stellte sich diese alte Frage neu. Sie sei ausgeführt, um die Diskussion über das Verständnis der Hanse in unserer Zeit anläßlich des 600. Jahrestages des Friedens von Stralsund herauszufordern und zu eröffnen.

In der Epoche des Stralsunder Friedens hatte der Prozeß eines tiefgreifenden ökonomisch-sozialen und politischen Umbruchs bereits eingesetzt, welcher die europäische Feudalgesellschaft in seinem Ergebnis in ein neues Entwicklungsstadium, aus dem Mittelalter in die Neuzeit führte. Strukturwandlungen in der 
Landwirtschaft, der gewerblichen Produktionssphäre wie der Zirkulationssphäre riefen Krisen hervor, führten zu Stillständen, Abbrüchen und Neuentwicklungen, die schließlich in die frühkapitalistische Entwicklung mündeten. Die sozialen und politischen Folgen betrafen alle mittelalterlich-feudalen Klassen, Stände und Schichten. So verschärften sich die sozialen Kämpfe wie die politischen und ideologischen Auseinandersetzungen. Der Feudalstaat trat in eine neue Umformung ein. Aus ihr gingen schließlich die deutschen Landeshoheiten und die großen, wesentlich auf Nationalität basierenden Monarchien Europas hervor, welche einerseits die mittelalterlichen Städte ihrer Autonomie beraubten und andererseits die bürgerlich-frükapitalistisch-bourgeoise Entwicklung förderten.

In den Beginn dieses Prozesses sahen sich die Hansekaufleute, die Inhaber des Zwischenhandelsmonopols im Nord- und Ostseeraum und des Stadtregiments in den Hansestädten, hineingestellt, als sich die Ausbildung der Städtehanse vollendet hatte und der Frieden von Stralsund geschlossen wurde. Die Städtehanse empfing neue Aufgaben, in der sie sich als „spezifische Organisationsform des Handelskapitals und als Instrument der in den Hansestädten herrschenden Schicht"1 zu bewähren, Aufgaben, denen die Kaufmannshanse nicht gegenübergestanden hatte. Sie sah sich konfrontiert dem Eindringen der auf eine neue Wirtschaftsweise gestützten und von einer starken Staatsgewalt geförderten holländischen und englischen Konkurrenz, einem aus der Veränderung der Wirtschaftslage und der Handelsbeziehungen genährten Sonderinteresse hansischer Städte, Bestrebungen des Territorialfürstentums, unter den Bedingungen des 15. Jahrhunderts die urbane Autonomie zu brechen, nicht zuletzt der sich im sozialen Differenzierungsprozeß steigernden bürgerlichen und plebejischen Opposition. Die wirtschaftlich-gesellschaftlichen Wandlungen und ihre politischen Konsequenzen bedrohten das Zwischenhandelsmonopol der Städtehanse, die Rolle Lübecks, der hansischen Führungsmacht, die Städtefreiheit sowie das Stadtregiment der aristokratisch-oligarchischen kaufmännischen Ratsgeschlechter.

Die Städtehanse verstand es, der Gefahren, Prüfungen, Rückschläge und Einbußen hindurch bis zum Ende des 15. Jahrhunderts Herr zu werden, bis in die Aera, in der der Frühkapitalismus in Europa durchbrach. Der Friede von Utrecht 1474 dokumentierte noch einmal ihre politische Machtstellung - wenn der Vertrag mit dem Herzog von Burgund und den Holländern insgesamt auch wenig günstig für die Hanse war, so gab sich England besiegt, unterwarf sich Köln und wurden in der Folge gute Beziehungen zu Frankreich hergestellt. Die Städtehanse hatte sich bis dahin im lübisch-hansestädtisch-handelskapitalistischen Interesse bewährt, gleich wie die Bewährung in den einzelnen Punkten zu bewerten ist.

1 Fritze, K., Am Wendepunkt der Hanse. Untersuchungen zur Wirtschafts- und Sozialgeschichte wendischer Hansestädte in der ersten Hälfte des 15. Jahrhunderts, Berlin 1967, S. 11. (Veröffentlichungen des Historischen Instituts der Ernst-Moritz-ArndtUniversität Greifswald, Bd. 3.) 
Der vom ökonomisch-sozialen Prozeß der Epoche mitsamt seinen politischen Konsequenzen und vom Klassencharakter sowie von der politischen Funktion der Städtehanse bestimmte Gedankengang führt an dieser Stelle zu der Frage, ob die Städtehanse, die als spezifische Organisationsform des lübisch-hansestädtischen Handelskapitals und als Instrument der in den Hansestädten herrschenden Ratsoligarchie handelte und keine Unterstützung durch eine historisch progressive Königsmacht erfuhr, unter den veränderten Existenzbedingungen mehr zu leisten vermochte. Bei einer Verneinung entsteht die Versuchung, die Geschichte der Hanse von der zweiten Hälfte des 14. bis zum letzten Viertel des 15. Jahrhunderts in einer Abwandlung des Titels, den Daenell seiner Darstellung dieses Zeitraums hansischer Geschichte gab ${ }^{2}$, als Blütezeit der Städtehanse anzusprechen.

Bei der Abwägung des pro und contra einer solchen Beurteilung wären für die deutsche Geschichte wesentliche Wirkungen hansischer Politik im 15. Jahrhundert zu bedenken. Schildhauer verweist auf die starken objektiven Impulse zur wirtschaftlichen Vereinigung des gesamten norddeutschen Raumes: „Der Beitritt einer großen Anzahl von binnenländischen Städten zur Hanse zeigt deutlich, daB sich in dieser Periode neben dem Seehandel der Überlandhandel bedeutend erweiterte und intensivierte. Er verband Nord- und Mitteldeutschland enger miteinander und begann Anschluß an das süddeutsche Wirtschaftsgebiet zu gewinnen. Die Tendenz zur Herausbildung eines einheitlichen Marktes ist hier unverkennbar." 3 Zugleich bestand die Wirkung der Städtehanse objektiv darin, daß für die Politik eines auf die Reichszentralisation und das Bündnis mit den Städten bedachten Königtums, für die „ständische (noch feudale, verwesend feudale und embryo-bürgerliche) Monarchie" ${ }^{4}$ relativ günstige Voraussetzungen, Möglichkeiten und Ansatzpunkte bestehen blieben bzw. geschaffen wurden. So schreibt Voigt: „Was die innerdeutsche Entwicklung betrifft, hat es (das hansische Bürgertum) bis ins 15. Jahrhundert durch seine überterritoriale Organisation die Konsolidierung der norddeutschen Fürstentümer erheblich gehemmt und damit eigentlich dem deutschen Königtum den Weg nach dem Norden offengehalten."5 Städtehanse sowie hansestädtische Einungen und Bündnisse unter Führung Lübecks bewiesen - bei allen Einschränkungen - von der Kölner Konföderation 1367 bis zum Frieden von Utrecht 1474 die Potenz des niederdeutschen Bürgertums, Fragen der politischen Vormacht im hansischen Einzugsgebiet zu seinen Gunsten zu entscheiden. Es hielt in dieser Zeit objektiv die Möglichkeit offen, als potentieller Bündnispartner eines auf die Nationalmonarchie, das Bündnis mit den Städten und die Beschränkung der

2 Dafnell, E., Die Blütezeit der deutschen Hanse. Hansische Geschichte von der zweiten Hälfte des 14. bis zum letzten Viertel des 15. Jahrhunderts, 2 Bde., Berlin 1905/06.

${ }^{3}$ Schildhader, J., unter Mitarbeit von K. Fritze, H. Langer, K. Spading, W. Stark, Grundzüge der Geschichte der deutschen Hanse, in: ZfG 11/1963, S. 738.

4 Engels, F., Zum „Bauernkrieg“, in: Marx/Engels, Werke, Bd. 21, Berlin 1962, S. 402.

5 Voigt, E., Reichsgewalt und Hansisches Bürgertum, in: WissZsGreifswald 12/1963, S. 516. 
Partikulargewalten bedachten Königtums, einen entsprechenden Beitrag in die Allianz zwischen Königtum und Bürgertum einzubringen.

Dabei verdient das hansestädtische Wirtschaftswachstum der späthansischen Zeit Beachtung. „Am Beispiel Wismars, Rostocks, Stralsunds, andeutungsweise Lübecks, Hamburgs und Danzigs“ demonstrierte Olechnowitz „eine allgemeine Konjunktur von Handel, Schiffahrt und gewerblicher Produktion 6". Die Untersuchungen von Fritze haben diesen Prozeß von der quantitativen Seite her im wesentlichen bestätigt. Wenn Fritze zugleich zu der Auffassung gelangt, daß eine nennenswerte frühkapitalistische Qualität nicht erreicht worden und die Wirtschaftsentwicklung im großen und ganzen in erstarrten, nicht in die Zukunft weisenden Formen verlaufen sei ${ }^{7}$, so sollte seine Einsicht nicht dazu verführen, die ökonomische Bedeutung der Handels-, Schiffahrts-, Schiffbauund Brauereikonjunktur wendischer Hansestädte zu unterschätzen bzw. als politischen Faktor nicht zu veransehlagen.

Was die Städtehanse im 15. Jahrhundert nicht vermochte und darum nicht in die Bewertungsmaßstäbe ihrer Leistung einbezogen werden dürfte, ist zweierlei : sie konnte zum ersten nicht die Rolle der Zentralgewalt ersetzen, und sie konnte ohne den monarchischen Integrationsfaktor sowie die monarchisch-nationalstaatliche Förderung schwerlich die nationalstaatliche Orientierung gewinnen. ${ }^{8}$ Zum zweiten konnte die Städtehanse die Veränderung der wirtschaftlich-gesellschaftlichen Wirklichkeit sowie die aus ihr resultierende Veränderung der ökonomischen Relationen und politischen Kräfteverhältnisse objektiv nicht verhindern, so daß ihre Existenz unabhängig vom subjektiven hansestädtischen Bemühen, gleich welcher Art, mit Notwendigkeit in Frage gestellt wurde. „Die privilegierte Stellung des hansischen Kaufmanns im Ausland verlor ihren Sinn in dem Augenblick,“ betont Olechnowitz, „als man ihn nicht mehr brauchte, entweder weil die eigene Wirtschaft schon stark genug war oder weil andere Wirtschaftsmächte aufrückten, die günstigere Bedingungen boten, als die Hansen zu geben bereit waren. Damit verlor die Hanse ihre progressive historische Stellung und ging ihrem notwendigen Ende entgegen." 9

Muß die entscheidende Ursache für den Auflösungsprozeß der Städtehanse darin gesehen werden, daß die Hanse, ,ungeachtet eines bemerkenswerten Aufschwungs von Handel und Schiffahrt, auf einer historisch überholten Stufe der sozialökonomischen Entwicklung stehengeblieben “ sei ${ }^{10}$, daß in den meisten Hansestädten an feudalen Produktionsformen festgehalten worden sei und diese den Gesamtcharakter der Produktion bestimmt hätten? Auch dieses

6 Olechnowitz, K.-F., Handel und Seeschiffahrt der späten Hanse, Weimar 1965, S. 182 f. (Abhandlungen zur Handels- und Sozialgeschichte, Bd. 6.)

7 Fritze, Wendepunkt, a. a. 0.

8 Müller-Mertens, E., Vom Regnum Teutonicum zum Heiligen Römischen Reich Deutscher Nation. Reflexionen über die Entwicklung des deutschen Staates im Mittelalter, in: ZfG 11/1963, S. $344 \mathrm{ff}$.

9 OLEchnowitz, Handel, a. a. O., S. 184.

10 Ebenda, S. 22. 
Problem möchte weiter durchdacht werden. Zur inhaltlichen Verdeutlichung der Frage sei unterstellt, unter den Hansestädten hätten Lübeck und eine Reihe anderer zum Kern der Hanse gehörender Städte ein leistungsfähiges Exportgewerbe entwickelt, das Handelskapital wäre in wesentlichem Maße in die Produktion eingedrungen, der Weg in die frühkapitalistische Entwicklung wäre eingeschlagen worden. Was hätte sich hieraus für das hansische Handels- und Verkehrssystem, die hansestädtischen Beziehungen von Reval, Dorpat und Riga über Danzig und Elbing bis Deventer, Kampen und Zwolle, die inneren Verhältnisse der den frühkapitalistischen Weg gehenden Hansestädte ergeben? Durchdenken wir auch eine noch weitergehende progressive Entwicklung. Was wäre geschehen, wenn zu einer sich eine eigene ökonomische Basis schaffenden wendischen Städtegruppe eine starke Staatsgewalt getreten wäre, wenn die Nationalmonarchie im niederdeutschen Raum Einzug gehalten und die sich dort - in Lübeck und in anderen Seestädten - entwickelnde Handelsbourgeoisie unterstützt hätte? Sollte die Städtehanse unter derartigen Umständen nicht zur Auflösung verurteilt gewesen sein und nicht ihr Ende gefunden haben? Eine progressive frühkapitalistische Entwicklung im Raum der wendischen Hansestädte mit oder ohne deren bzw. dessen Integration in eine Nationalmonarchie, einen werdenden absoluten Staat hätte Konflikte beschworen und Interessen gesetzt, Relationen hergestellt und Realitäten geschaffen, die der Städtehanse - so wie ihr Wesen und ihr Klassencharakter beschaffen waren vermutlich einen früheren Schlußpunkt gesetzt, das heißt sie früher aufgelöst hätten, als es die tatsächlichen geschichtlichen Umstände taten.

Jedenfalls hätte die Hanse ihr Wesen und ihren Klassencharakter grundsätzlich wandeln, historisch überlebte Wesenszüge aufgeben, neue, den sich verändernden Bedingungen entsprechende gewinnen, sich auf einen Kern territorialstaatlich, wohl auch produktionsmäßig verbundener Hansestädte einengen müssen. In einem solchen Prozeß hätte die Städtehanse aufgehört, als das zu existieren, was sie ihrem Wesen nach war, sie wäre von neuen Kräften in ihrem Interesse umfunktioniert worden, wäre in einem inhaltlich neuen Interessenverband aufgehoben worden. Das Zwischenhandelsmonopol eines nicht auf eine einheimische Produktion gestützten mittelalterlichen Handelsbürgertums, die Autonomie der aus dem feudalherrlichen Staatsverband herausgelösten Bürgerkommune, das Stadtregiment aristokratisch-oligarchischer fernhändlerischer Ratsgeschlechter zu verteidigen, darin hätten die Hauptfunktionen fürderhin nicht mehr bestehen können. Aus der Städtehanse des mittelalterlichen hansestädtischen Handelsbürgertums hätte sich entwickeln müssen ein Instrument einer auf eine eigene Produktionsbasis und eine frühkapitalistische Entwicklung in der Produktion gestützten sowie dem Staat eingegliederten Handelsbourgeoisie und einer frühabsolutistischen (ständischen, noch feudalen, verwesend feudalen und embryo-bürgerlichen) Monarchie.

Das Eingehen auf dieses Problem, das hier in heuristischer Absicht gestellt ist, erfordert eine weitere und vertiefte Verständigung über das Wesen der Hanse. Dazu sind in jüngster Zeit von marxistischen Hansehistorikern wesent- 
lich weiterführende Beiträge geleistet worden. Die Hansehistoriographie auf historisch-materialistischer Grundlage arbeitete heraus, daß die Hanse primär von ihrem Klassencharakter zu begreifen ist. ${ }^{11}$ Doch sind noch weitere Forschungen nötig, um zu einer voll befriedigenden Bestimmung des Wesens der Hanse zu kommen. Besondere Aufmerksamkeit verdient die Forderung von Fritze, eine umfassende vergleichende Untersuchung mit den mittelalterlichen Städtebünden anzustellen.12 Zugleich wäre eine eindringliche Struktur- und Funktionsanalyse der Hanse, ihrer Elemente und Relationen, eine Analyse der Hanse als offenes dynamisches System in Angriff zu nehmen.

Diese Fragen zu stellen - sie stehen stellvertretend für andere - gibt der Tag Anlaß, an dem vor 600 Jahren die Hanse in Stralsund mit Dänemark Frieden schloß und ihren Höhepunkt erreichte. Das säkulare Ereignis fordert das weiterführende Gespräch über die Hanse und die Hanseaten heraus. Angesichts der wissenschaftlichen und politischen Aktualität der hansisch-hanseatischen Grundfragen werden Hanse- und Städtehistoriker, wie Vertreter der Wirtschafts-, Sozial- und politischen Geschichte anläßlich des Jubiläums in eine internationale Diskussion über die Hanse eintreten, die gleichermaßen von Interesse für die Geschichte des deutschen Volkes sein wird wie für die Geschichte der Ost- und Nordseeländer, die allgemeine Handels-, Wirtschafts- und Seefahrtsgeschichte und die vergleichende Betrachtung.

In die internationale wissenschaftliche Diskussion Ergebnisse der eigenen Arbeit hineinzustellen, ist der hansischen Arbeitsgemeinschaft in der Deutschen Demokratischen Republik ein Bedürfnis und bewegt sie, ihre Neuen Hansischen Studien in Verbindung mit dem 600. Jahrestag des Stralsunder Friedens herauszubringen.

Die Neuen Hansischen Studien sind insbesondere erwachsen aus dem wissenschaftlichen Ertrag der Arbeitstagungen von Wismar 1966, Görlitz 1967 und Rostock 1968, dem wachsenden Austausch mit polnischen und sowjetischen Historikern in diesen Jahren sowie der in Greifswald verankerten vorbereitenden Arbeit zu einer marxistischen Gesamtdarstellung der Hansegeschichte. Die Neuen Hansischen Studien stehen in Entsprechung zu dem 1961 erschienenen, Heinrich Sproemberg zum 70. Geburtstag gewidmeten Band Hansische Studien, welche den Ertrag der Arbeitstagungen in Leipzig, Schwerin, Stendal und Berlin 1956-1959 spiegeln.13

Seit dem Erscheinen der Hansischen Studien im Jahre 1961 ist auf dem Gebiet der Hansegeschichte in der DDR weitere wesentliche Arbeit geleistet

11 Schildhader, J., Progressive und nationale Traditionen in der Geschichte der Hanse, in: WissZsGreifswald 12/1963, S. 500; Ders., Grundzüge, a. a. O.; OLechNowitz, Handel, a. a. O., S. 6f.; Fritze, Wendepunkt, a. a. O., S. 11; Stern, L. und E. Vorgt, Deutschland in der Feudalepoche von der Mitte des 13. Jh. bis zum ausgehenden 15. Jh. Lehrbuch der deutschen Geschichte (Beiträge), Bd. 2/3, Berlin 1964, bes S. 56-66, 189-195.

12 Fritze, Wendepunkt, a. a. O., S. 12.

13 Hansische Studien. Heinrich Sproemberg zum 70. Geburtstag, Berlin 1961. (Forschungen zur mittelalterlichen Geschichte, Bd. 8.) 
worden. In diesem Zeitraum führte die Arbeitsgemeinschaft die Hanseund Städtehistoriker, zahlreiche an hansisch-hanseatischer Geschichte interessierten Archivare, Bibliothekare, Kommunalpolitiker, Lehrer, Museologen und Studenten sowie andere Interessenten auf acht wissenschaftlichen Tagungen zu gemeinsamer Arbeit, zur Vermittlung und Verbreitung neuer Forschungsergebnisse, zur Orientierung auf aktuelle Probleme und zur Diskussion zusammen. Die Arbeitstagungen wurden mit wachsender internationaler Beteiligung durchgeführt und gewannen zuletzt den Charakter internationaler Konferenzen. 1961 in Naumburg standen Fragen der sozial-ökonomischen und politischen Auseinandersetzungen in den spätmittelalterlichen Städten zur Diskussion. ${ }^{14}$ Die Rostocker Arbeitstagung 1962 ging der Stellung der Hanse in der deutschen Geschichte nach. ${ }^{15}$ Den Wirtschaftsbeziehungen der Hanse zu ihrem nord- und mitteldeutschen Hinterland wandte sich 1963 die Schweriner 16, Problemen der Handelsgeschichte 1964 die Leipziger Jahresversammlung ${ }^{17} \mathrm{zu}$. In Wismar 1966 konzentrierte sich die Arbeitsgemeinschaft auf die hansischen Quellen aus dem städtischen Bereich. Es galt, sich vertiefter über ihre Aussagekraft unter dem Gesichtspunkt der Sozial-, Handels- und Verkehrsgeschichte zu verständigen. ${ }^{18}$ Die städtischen Volksbewegungen in den Hansestädten zur Zeit von Reformation und Bauernkrieg standen 1967 im Mittelpunkt der Görlitzer Tagung. Die Arbeitsgemeinschaft leistete damit ihren Beitrag zur Vorbereitung des 450. Jahrestages der Reformation. ${ }^{19}$ Zur 750. Jahrfeier Rostocks 1968 wurde wieder in dieser Stadt getagt, um zum Jubiläum der Stadtrechtsbestätigung neue Forschungsergebnisse über das hansische Rostock und seine internationalen Beziehungen vorzustellen. ${ }^{20}$ Als Beitrag zum 20. Jahrestag der DDR gestaltete die Arbeitsgemeinschaft ihre Mühlhäuser Tagung 1969, auf der die „Änderungen der Handelswege und Handelsstruktur beim Übergang vom Mittelalter zur Neuzeit" (unter besonderer Beachtung der Rolle der Wirtschaftsveränderungen) im Mittelpunkt der Beratungen standen.21

14 LAUBe, A., 7. Arbeitstagung der DDR-Arbeitsgemeinschaft des Hansischen Geschichtsvereins, in: ZfG 10/1962, S. 184-188.

${ }^{15}$ LAUBE, A., 8. Arbeitstagung der DDR-Arbeitsgemeinschaft des Hansischen Geschichtsvereins in Rostock, in: ZfG 11/1963, S. 395-398.

${ }^{16}$ LAUBE, A., 9. Arbeitstagung der DDR-Arbeitsgemeinschaft des Hansischen Geschichtsvereins in Schwerin, in: ZfG 12/1964, S. 95-99.

17 VoGLER, G., 10. Arbeitstagung der DDR-Arbeitsgemeinschaft des Hansischen Geschichtsvereins in Leipzig, in: ZfG 13/1965, S. 306-309.

18 ENGEL, E., und S. Looss, 11. Arbeitstagung der DDR-Arbeitsgemeinschaft des Hansischen Geschichtsvereins, in: ZfG 15/1967, S. 304-307.

19 VeTter, K., 12. Arbeitstagung der Arbeitsgemeinschaft des Hansischen Geschichtsvereins in der DDR, in: ZfG 16/1968, S. 215-217.

20 Plöse, D. und K. Vetter, 13. Jahrestagung der Arbeitsgemeinschaft des Hansischen Geschichtsvereins in der DDR in Rostock, ZfG in: 17/1969. S. 625-627.

21 VETTER, K., 14. Jahrestagung der Arbeitsgemeinschaft des Hansischen Geschichtsvereins in der DDR, in ZfG: 18/1970, erscheint in Heft 2. 
Die Arbeitstagungen förderten die internationalen Kontakte, insbesondere die Zusammenarbeit mit den Historikern der sozialistischen Länder; der hansische Austausch zwischen Universitäten der DDR und anderer sozialistischer Länder wurde durch gegenseitige Gastvorlesungen und Vortragsreisen erheblich verstärkt. Mit der Gdańskie Towarzystwo Naukowe, Gdańsk, schloß die Arbeitsgemeinschaft Vereinbarungen über regelmäßige Besuche ab.

Dazu stellt sich eine rege Publikationstätigkeit auf dem Gebiet hansischer Geschichte.22 Eine besondere Rolle spielen Untersuchungen zur Sozial- und Wirtschaftsgeschichte der späthansischen Zeit, insbesondere der wendischen Hansestädte. Neben Aufsätzen und Diskussionsbeiträgen stehen hier gewichtige Monographien wie die Bücher von Fritze und Olechnowitz, die zu grundsätzlich neuen Einsichten in das Wesen der Hanse gelangten. ${ }^{23}$ Diese Forschungen werden in Hinsicht auf die Erarbeitung eines Gesamtbildes ergänzt durch Arbeiten über die Verbindung der Seestädte mit dem agrarischen Hinterland, über den Getreidehandel, das Bürgerkapital und das Lehnbürgertum sowie über Volksbewegungen in einzelnen Hansestädten. ${ }^{24}$ Dazu kommen Studien über die inter-

$22 \mathrm{Zu}$ den Publikationen bis 1960 bzw. 1962 : K. Fritze, E. MÜLler-Mertens, J. SchildHAURR und M. UNGER, Forschungen zur Stadt- und Hansegeschichte in der DDR, in: Historische Forschungen in der DDR, ZfG 7/1960, S. 74-104; ScHWDHAUER, J., Forschungen zur hansischen und hanseatischen Geschichte 1960-1962, in: WissZsGreifswald 12/1963, S. 129 - 146. Als neue regionalgeschichtliche Publikationsorgane aus dem Hanseraum erscheinen seit 1961 das "Greifswald-Stralsunder Jahrbuch" (herausgegeben von dem Kulturhistorischen Museum Stralsund, dem Stadtarchiv Stralsund, dem Landesbzw. Staatsarchiv Greifswald, dem Museum der Stadt Greifswald und dem Stadtarchiv Greifswald) und seit 1966 die „Rostocker Beiträge. Regionalgeschichtliches Jahrbuch der mecklenburgischen Seestädte" (herausgegeben von den Stadtarchiven Rostock und Wismar durch J. LACHs).

23 Fritze, K., Der Kampf um die Demokratisierung des Stadtregiments in Wismar 1427 bis 1430, in: WissZsGreifswald 13/1964, S. 249-258; DERs., Die Bevölkerungsstruktur Rostocks, Stralsunds und Wismars am Anfang des 15. Jahrhunderts. Versuch einer sozialstatistischen Analýse, in: GreifswStralJb 4/1964, S. 69-79; DERs., Einige Bemerkungen zum Problem der hansischen Handelsprofite im 14. und 15. Jahrhundert, in: WissZsGreifsw 14/1965, S. 245-248; DERs., Keimformen der kapitalistischen Produktionsweise in wendischen Hansestädten zu Beginn des 15. Jahrhunderts, in: JbWG 1965, Teil 4, S. 193-209; DERS., Zur Lage der hansestädtischen Plebejer, in: RostBeitr 1/1966, S. 41-44; DERs., Stralsunds Bevölkerung um 1400, in: GreifswStralJb 6/1966, S. 15-28; DERS., Wendepunkt, a. a. 0.; LANGER, H., Zur Rolle der Lohnarbeit im spätmittelalterlichen Zunfthandwerk der Hansestädte, in Jb f. Regionalgeschichte 3/1968, S. 92108; Neuss, E., Hanse und niedersächsische Städtebünde in ihrem Verhältnis zu den sozialen Bewegungen im Elbe-Saale-Raum während des Spätmittelalters, in: JbRegionalg 1/1965, S. 153-163; Olechnowitz, Handel, a. a. O.; Winter, H., Das Hanseschiff im ausgehenden 15. Jahrhundert, Rostock 1961.

24 Assing, H., Die Eigentums- und Herrschaftsverhältnisse in den Dörfern des Teltow in der Zeit um 1375, phil. Diss. Berlin 1965 (MS.); Czok, K., Bürgerkämpfe und Chronistik im deutschen Spätmittelalter. Ein Beitrag zur Herausbildung bürgerlicher Geschichts- 
nationalen hansischen Verbindungen, die Handelsgeschichte und die politischen Beziehungen. ${ }^{25}$ Eine besondere Vortragsfolge und Aufsatzreihe zur hanseatischen Geschichte widmet sich den Beziehungen der Hansestädte zu Lateinamerika im

schreibung, in: ZfG 10/1962, S. 637 - 645; DERS., Kommunale Bewegung und bürgerliche Opposition in Deutschland im 13. Jahrhundert, in: WissZsLeipzig 14/1965, S. 413 bis 418; Donnert, E., Bemerkungen zur Frage der Reformation und der Volksbewegung in Livland, in: Die frühbürgerliche Revolution in Deutschland, Berlin 1961, S. 145-151 (DHG, Tagung der Sektion Mediävistik 1960 in Wernigerode, Bd. 2). Engel, E., Bürgerlicher Lehnsbesitz, bäuerliche Produktenrente und altmärkisch-hamburgische Handelsbeziehungen im 14. Jahrhundert, in: HGbll 82/1964, S. 21-41; Engel, E., und B. Zientara, Feudalstruktur, Lehnbürgertum und Fernhandel im spätmittelalterlichen Brandenburg, mit einer Einleitung von E. Müller-Mrrtens, Weimar 1967 (Abhandlungen zur Handels- und Sozialgeschichte, Bd. 7.); Fritze, K., Probleme der Stadt-Land-Beziehungen im Bereich der wendischen Hansestädte nach 1370, in: HGbll 85/1967, S. 38-58; KuHLEs, J., Studien zur sozialen Lage der Volksmassen und zu den Volksbewegungen zur Zeit der Reformation in Livland, phil. Diss. Leipzig 1966 (MS); DERs. und W. Küttler, Bürgertum und Reformation in den ostbaltischen Ländern, in: 450 Jahre Reformation, hrsg. von L. Stern und M. Steinmetz, Berlin 1967 S. 186-200; Kürtler, W., Patriziat, Bürgeropposition und Volksbewegung in Riga in der zweiten Hälfte des 16. Jahrhunderts, phil. Diss. Leipzig 1966 (MS.); MüLleRMertens, E., Berlin und die Hanse, in: HGbll 80/1962, S. 1-25; Ders., Fritz Rörig, das Landbuch Karls IV. und das märkische Lehnbürgertum, in: EnGEL/Zientara, Feudalstruktur, a. a. O., S. 1-28; SAMSONOwICz, H., Untersuchungen über das Dainziger Bürgerkapital in der zweiten Hälfte des 15. Jahrhunderts, Ubersetzung aus dem Polnischen, Weimar 1969. (Abhandlungen zur Handels- und Sozialgeschichte, Bd. 8.) Die Arbeitsgemeinschaft förderte diese Thematik durch die Ubersetzung der genannten Werke von Samsonowicz und ZIENTARA, sie förderte die vergleichende Betrachtung durch die Herausgabe des Werkes UnGER, M., Stadtgemeinde und Bergwesen Freibergs im Mittelalter, Weimar 1963 (Abhandlungen zur Handels- und Sozialgeschichte, Bd. 5.) und den Goslarer Vortrag von LAOBE, A., Bergbau, Bergstädte und Landesherrschaft im 15./16. Jahrhundert, in: ZfG 16/1968, S. 1577-1591.

25 DonNERT, E., Die Hanse und Rußland in den Jahren des Livländischen Krieges 1558-83, in: WissZsLeipzig 14/1965, S. 511-516; FrITZE, K., Dänemark und die hansisch-holländische Konkurrenz in der Ostsee zu Beginn des 15. Jahrhunderts, in: WissZsGreifsw 13/1964, S. 79-87; Gringmuth-Dallmer, H., Magdeburg - Haupthandelsplatz der mittleren Elbe, in: HGbll 84/1966, S. 8-19; LANGER, H., Stralsunds Entscheidung 1628, in: GreifswStralJb 4/1964, S. 81-98; DERS., Die Rolle Stralsunds bei der Vorbereitung und beim Beginn der schwedischen Aggression in Deutschland 1630, in: WissZsGreifswald 15/1965, S. 253-263; ReINHold, J., Die Leipziger Messen und Polen/Litauen in der zweiten Hälfte des 18. Jahrhunderts, phil. Diss Rostock 1967 (MS); ScHILDHAdER, J., Zur Verlagerung des See- und Handelsverkehrs im nordeuropäischen Raum während des 15. und 16. Jahrhunderts. Eine Untersuchung auf der Grundlage der Danziger Pfahlkammerbücher, in: JbWG 1968, Teil 4, S. 187-211; UnGER, M., Die Leipziger Messe und die Niederlande im 16. und 18. Jahrhundert, in: HGbll 81/1963, S. 1-19; ZokLLNER, K. P., Zu den hansisch-norwegischen Beziehungen am Ausgang des Mittelalters (1550 bis 1600), in: Nordeuropa, Studien II, WissZsGreifsw 16/1967, S. 115-127. 
19. und zu Beginn des 20. Jahrhunderts.26 $\mathrm{Zu}$ nennen sind ferner Quellenpublikationen und quellenkundliche Studien ${ }^{27}$, nicht zuletzt Monographien zur Geschichte einzelner Hansestädte. ${ }^{28}$ Für ein breites Publikum gedacht, die Ergebnisse der Hanseforschung popularisierend, haben diese besondere Bedeutung als massenwirksame historische Literatur. In der Gegenüberstellung von spezieller quellenkritischer Arbeit und allgemeinverständlicher Städtemonographie wird die Verbindung von strenger Quellenforschung und populärer Verbreitung wissenschaftlicher Erkenntnisse in der hansischen Arbeit sichtbar.

Dazu wurde über die genannten Arbeiten hinaus in eine Diskussion über die Stellung der Hanse in der deutschen Geschichte und das Wesen der Hanse eingetreten $^{29}$, wurde ein Meinungsstreit über die Grundzüge der Geschichte der deutschen Hanse aufgenommen, wurde zugleich eine Reihe von Dissertationen über hansische Strukturfragen des 15. und 16. Jahrhunderts angefertigt ${ }^{30}$, sind

${ }^{26}$ KATZ, F., Hamburger Schiffahrt nach Mexiko 1870-1914, in: HGbll 83/1965, S. 94-108; Kоssок, M., Bremen, Preußen und die „Texas-Frage“ 1835-1846, in: BremJb 49/1964, S. 73-104; DERS., Zur Geschichte der deutsch-lateinamerikanischen Beziehungen (Forschungs- und Periodisierungsprobleme), in: HGbll 84/1966, S. 49-77; Zeuske, M., Die Hansestädte und das Reich in Venezuela zur Zeit des Venezuelakonflikts, Vortrag auf der 83. Jahresversammlung des HGV 1967 in Soest.

27 Der Stralsunder Liber memorialis, bearbeitet von H.-D. Schroeder, Teil 1: Fol. 1-60. 1320-1410, Schwerin 1964, Teil 4: Fol. 187-240. 1366-1426, Rostock 1966 (Veröffentlichungen des Stadtarchivs Stralsund, Bd. 4, 1 und 4.); Das zweite Wismarsche Stadtbuch 1272-1297, bearbeitet von L. KNaBE unter Mitwirkung von A. DüsING, 2 Bde. (Text und Register), Weimar 1966 (Quellen und Darstellungen zur hansischen Geschichte Neue Folge, Bd. 14, 1 und 2.); Hansische Handelsstraßen, auf Grund von Vorarbeiten von F. Bruhns ( $\dagger$ ) bearbeitet von H. WrCzERKa, Registerband, bearbeitet von E. ENGEL und H. Weczerka unter Mitarbeit von J. Bongard, Weimar 1968 (Quellen und Darstellungen zur hansischen Geschichte Neue Folge, Bd. 13, 3.); ScHLdHaUER, J., Hafenzollregister des Ostseebereiches als Quellen zur hansischen Geschichte, in: HGbll 86/1968, S. 63-76; StakK, W., Die Danziger Pfahlkammerbücher (1468-1476) als Quelle für den Schiffs- und Warenverkehr zwischen den wendischen Hansestädten und Danzig, in: RostBeitr 1/1966, S. 57-78.

28 Ewe, H., Stralsund, Schwerin 1962, 2. Aufl., Rostock 1965; Fritze, K., Die Hansestadt Stralsund. Die beiden ersten Jahrhunderte ihrer Geschichte, Schwerin 1961; (Veröffentlichungen des Stadtarchivs Stralsund, Bd. 4.) LACHS, J., und F. K. RAIF, Rostock, Rostock 1965; OLECHNOwITZ K.-F., Rostock von der Stadtrechtsbestätigung im Jahre 1218 bis zur bürgerlich-demokratischen Revolution von 1848/49, Rostock 1968. (Beiträge zur Geschichte der Stadt Rostock, Bd. 1.) Vermerkt sei auch EwE, H., Rügen, Rostock 1966.

29 Schildhaukr, Grundzüge, a. a. 0., S. 729-746, Abdruck auch in: WissZsGreifswald 14/1965, S. 195-203; Fritze, K., Tendenzen der Stagnation der Hanse nach 1370, in: WissZsGreifswald 12/1963, S. 519-524; Ders., Wendepunkt, a. a. O., S. 8-12; OLechNowITz, Handel, a. a. O., S. 3-9; Schildhader, Traditionen, a. a. O.; Voigt, Reichs. gewalt, a. a. 0., S. 507-517.

30 LANGer, H., Wirtschaft und Politik in Stralsund von 1600-1630, phil. Diss. Greifswald 1965 (MS.); Spading, K., Holland und die wendischen Hansestädte in der zweiten Hälfte 
weitere in Arbeit gegeben und geplant. Allen diesen Arbeiten ist zugleich die Auseinandersetzung mit der bürgerlichen-imperialistischen Geschichtsschreibung der Vergangenheit wie der Gegenwart eigen.

Die Neuen Hansischen Studien bieten einen Ausschnitt aus dieser Arbeit. Sie wollen im Zusammenhang mit der Gesamttätigkeit und ihren Zielen gesehen werden und sind insofern repräsentativ, als sie Beiträge zu Schwerpunkten der Diskussion hansischer Geschichte bieten. Die erste Serie ist den Quellen und der Historiographie gewidmet, der marxistischen Aneignung der Quellen, der Ausarbeitung marxistischer Methoden der Quellenkritik und der Auseinandersetzung mit der bürgerlichen Geschichtsschreibung. Die städtischen Volksbewegungen, den sozialgeschichtlichen Aspekt, die Rolle der Produktion und die bürgerlichen Feudalbeziehungen in der hansestädtischen Geschichte in ihrer Bedeutung zu erfassen und zu würdigen, für dieses Anliegen steht die zweite Serie: Stadtgeschichte und städtische Volksbewegungen. Der dritte Themenkreis „Handelsgeschichte und internationale Verbindungen“ erwächst aus der besonderen Pflege der internationalen Beziehungen. Es werden Entwicklungen im hansischen Handelssystem abgetastet, seit die Holländer in ihm als größte Sprengkraft erschienen, und werden die Verbindungen zu Preußen, Livland und Rußland sowie zum westeuropäischen und iberischen Bereich verfolgt. Gefördert werden Fragen, die der weiteren theoretischen Diskussion, der Erforschung und Auseinandersetzung bedürfen: die Frage nach der Definition der Hanse; die Frage nach ihrer Bedeutung für die Geschichte des deutschen Volkes; die Frage nach der Rolle der Hanse in der Geschichte des europäischen Städtewesens und Bürgertums sowie die Frage nach ihrer Einordnung in die europäische und allgemeine Geschichte.

des 15. Jahrhunderts, phil. Diss. Greifswald 1968 (MS.); Stark, W., Lübeck und Danzig in der zweiten Hälfte des 15. Jahrhunderts, phil. Diss. Greifswald 1969; (MS.) Zöllner, H. P., Studien zur Hansegeschichte der zweiten Hälfte des 16. Jahrhunderts unter besonderer Berücksichtigung der Stadt Stralsund, phil. Diss. Greifswald 1967 (MS.). 
